\title{
SUPERRESOLUTION SAR IMAGING ALGORITHM BASED ON MVM AND WEIGHTED NORM EXTRAPOLATION
}

\author{
P. Zhang ${ }^{\mathrm{a},}{ }^{*}$, Q. Chen ${ }^{\mathrm{a}}$, Z. Li ${ }^{\mathrm{a}}$, Z. Tang ${ }^{\mathrm{b}}$, J. Liu ${ }^{\mathrm{b}}$, L. Zhao ${ }^{\mathrm{b}}$ \\ ${ }^{\text {a }}$ Center for Earth Observation and Digital Earth Chinese Academy of Sciences \\ No.9 Dengzhuang South Road, Haidian District, Beijing 100094, China \\ ${ }^{\mathrm{b}}$ Beijing Institute of Spacecraft System Engineering,CAST, Beijing, 100094,China \\ E-mail: pzhang@ceode.ac.cn
}

KEY WORDS: Synthetic Aperture Radar (SAR), Superresolution, Minimum Variance Method, Minimum Weighted Norm, Extrapolation

\begin{abstract}
:
In this paper, we present an extrapolation approach, which uses minimum weighted norm constraint and minimum variance spectrum estimation, for improving synthetic aperture radar (SAR) resolution. Minimum variance method is a robust high resolution method to estimate spectrum. Based on the theory of SAR imaging, the signal model of SAR imagery is analyzed to be feasible for using data extrapolation methods to improve the resolution of SAR image. The method is used to extrapolate the efficient bandwidth in phase history field and better results are obtained compared with adaptive weighted norm extrapolation (AWNE) method and traditional imaging method using simulated data and actual measured data.
\end{abstract}

\section{INTRODUCTION}

One of the driving forces in the development of SAR image formation has been to obtain better and better image resolution. Conventional radar imaging methods based on Fourier transform provide good resolution as long as the backscattered data is available over a large bandwidth and a sufficient aspect region (Curlander, 1991). Wider transmitted bandwidth achieves higher range resolution. Either a higher center frequency or wider aspect angle variation will improve azimuth resolution. But in many practical applications only limited frequency and aspect bands are available. This leads to radar images with limited resolution. Recently, modern spectral estimation technologies have become a kind of new methods to enhance the image quality by obtaining better space resolution and lower sidelobe. Several modern spectral estimation methods are discussed by DeGraaf (DeGraaf, 1998) to enhance resolution of SAR, including adaptive sidelobe reduction (ASR), minimum variance method (MVM), and autoregressive (AR) model, etc. Improvements in image are shown in results, including resolution of prominent scatterers, and CFAR detectability of targets in clutters, etc. An one-dimensional (1-D) adaptive weighted norm extrapolation (AWNE) method is brought forward by Cabrera (1991), which has been used in ISAR (1994) and spotlight SAR (Barbarossa, 1996). Two-dimensional (2-D) AWNE method is put forward by Brito (Brito, 1999) used in spotlight SAR. This kind of AWNE is the iterative method and proved efficient in SAR resolution enhancement.

The paper provides an efficient bandwidth extrapolation algorithm to reach higher resolution in SAR imaging, which is based on minimum weighted norm constraint and minimum variance spectrum estimation. Unlike AWNE, the method in this paper exploits no iteration. The detailed derivation is given about the new method and better results prove the validation of the new method, compared with the results of AWNE and traditional algorithms.

The remainder of this paper is organized as follows. The signal model is presented in Section II. The detailed derivation is described in Section III. Numerical and experimental results are provided in Section IV. Finally, Section V concludes the whole paper.

\section{SIGNAL MODEL}

Based on the theory of synthetic aperture radar imaging, the signal model of SAR imagery is analyzed to be feasible by using data extrapolation methods to improve SAR image resolution in this section. Generally, SAR systems use linear FM signal as transmitted signal. The geometrical relationship of SAR sensor and target can be described as Fig.1 under the strip-map SAR condition. 


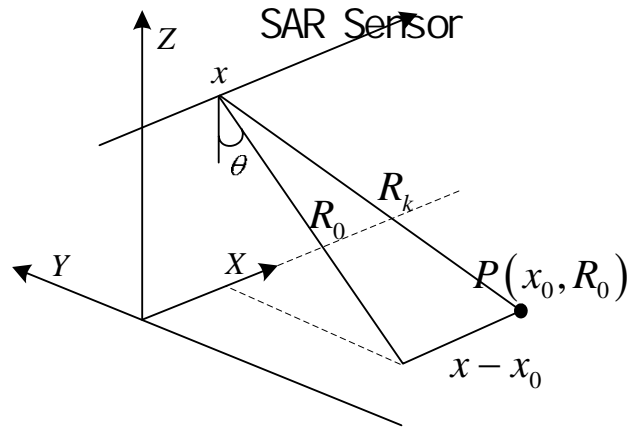

Fig.1. geometrical relationship of SAR sensor and target

After imaging processing, the form of scene echo can be represented (Curlander, 1991) as

$$
\begin{aligned}
s\left(t_{r}, t_{a}\right)= & \sum_{n=1}^{N} \sigma_{n} \operatorname{Sinc}\left[B_{r}\left(t_{r}-t_{1}\left(x_{r, n}\right)\right)\right], \\
& \times \operatorname{Sinc}\left[B_{a}\left(t_{a}-t_{2}\left(x_{a, n}\right)\right)\right]
\end{aligned}
$$

where $t_{r}$ and $t_{a}$ represent time in slant range and azimuth direction, respectively; $x_{r, n}$ and $x_{a, n}$ represent targets location in slant range and azimuth direction, respectively; $B_{\mathrm{r}}$ and $B_{a}$ represent transmit signal bandwidth and Doppler bandwidth in azimuth, respectively; $N$ is the number of scatter centers. Transforming (1) into phase history domain, we can see a rectangle support domain in the following expression

$$
\begin{aligned}
s\left(\omega_{r}, \omega_{a}\right)= & \sum_{n=1}^{N} \sigma_{n} \operatorname{Re} c t\left[\frac{\omega_{r}}{B_{r}}\right] \otimes \operatorname{Rect}\left[\frac{\omega_{a}}{B_{a}}\right] \\
& \times \exp \left(-j t_{1}\left(x_{r, n}\right) \omega_{r}\right) \times \exp \left(-j t_{2}\left(x_{a, n}\right) \omega_{a}\right)
\end{aligned}
$$

From the expression, we can see that SAR image signal model in phase history domain is a band-pass function with a main frequency support domain. Thus, the problem of superresolution SAR imaging is transformed to solve the efficient bandwidth extrapolation.

\section{METHODOLOGY}

The paper proposes a data extrapolation method based on minimum weighted norm constraint and minimum variance spectrum estimation, which is classified as nonparameter method. The method extrapolates the efficient bandwidth in phase history field. In this section, the detailed description of the method is given.

\section{A. New Extrapolation Method of 1-D Case}

Define data vector $\mathbf{x}$ as

$$
\mathbf{x}=[x(0) \cdots x(N-1)]^{\mathrm{T}} \text {. }
$$

A very general $L \times 1$ data vector denotes the observed continuous discrete-time data samples, i.e.

$$
\mathbf{y}=\left[x\left(m_{1}\right) \cdots x\left(m_{L}\right)\right]^{\mathrm{T}},
$$

where $\left\{m_{\mathrm{k}}, k=1 \cdots L\right\}$ is continuous integers and $\mathbf{y}$ is the observed part of the whole data vector $\mathbf{x}$.

The observed data can be expressed by the whole data as

$$
\mathbf{y}=\mathbf{T x},
$$

where each row of $\mathbf{T}$ is a vector of zeros with a nonzero elements 1 in observed position $m_{\mathrm{k}}$. Thus the problem of extrapolation can be described to be an inverse problem using the observed data $\mathbf{y}$ to solve the whole data $\mathbf{x}$.

An optimal unique choice $\{\hat{x}(n)\}_{n=0}^{N}$ is the Minimum Weighted Norm constraint (Potter, 1989; Cabrera, Sergio D, 1991; Brito, 1999), which solves

$$
\min _{\mathbf{y}}\left\{\int_{f \in B} \frac{|X(f)|^{2}}{P(f)} d f\right\}=\|\hat{x}(n)\|^{2}=\int_{f \in B} \frac{|\hat{X}(f)|^{2}}{P(f)} d f .
$$

The extrapolated $\hat{x}(n)$ is the result of an optimal spectral shape match of $|X(f)|^{2}$ to $P(f)$. B is the spectral support scope, i.e. $[-0.5,0.5]$. Let $P(f)>0, f \in B$, and $P(f)=0, f \notin B . P(f)$ is a prior frequency weighting function, i.e. estimated spectrum from the observed data. Then, we briefly introduce Capon's minimum variance method [2] to form the frequency weighting function $P(f)$ using the observed data $\left\{x\left(m_{1}\right) \cdots x\left(m_{L}\right)\right\}$.

Since the output consists of desired and undesired energy, and since the signal passes with unit gain, MVM maximizes SIR by selecting to minimize the expected output energy with the constraint to insure that the signal returned from interesting scatterers is passed with unit gain

$$
\min \left(\mathbf{h}_{\omega}^{\mathrm{H}} \mathbf{R} \mathbf{h}_{\omega}\right) \quad \text { subject to } \quad \mathbf{h}_{\omega}^{\mathrm{H}} \mathbf{a}(\omega)=1,
$$

where $\mathbf{h}_{\omega}$ is the complex-valued space-variant weighting vector; $\mathbf{R}$ is the covariance matrix, which is obtained with the forward covariance matrix using the forward observation vector and the backward covariance matrix using the backward observation vector to obtain robust estimation; $\mathbf{a}(\omega)=\left[\begin{array}{llll}1 & e^{j \omega} & \cdots & e^{j(p-1) \omega}\end{array}\right]^{\mathrm{T}}$.

The optimal filter is

$$
\mathbf{h}_{\omega}^{\mathrm{MVM}}=\frac{\mathbf{R}^{-1} \mathbf{a}(\omega)}{\mathbf{a}^{\mathrm{H}}(\omega) \mathbf{R}^{-1} \mathbf{a}(\omega)}
$$

and the minimum variance spectrum[1] is 


$$
P_{M V M}(\omega)=\frac{1}{\mathbf{a}^{\mathrm{H}}(\omega) \mathbf{R}^{-1} \mathbf{a}(\omega)} .
$$

When the power spectrum of the observed data samples is obtained, the greater scale data can be achieved using exploration with minimum norm weighted constraint.

In order to obtain $\{\hat{x}(n), n=0 \cdots N-1\}$, we should solve (4) subjecting to constraint (3) using the $P_{M V M}$ as the prior frequency weighting function. This is an inverse problem in Hilbert space, so the minimum norm solution can be expressed as

$$
\hat{\mathbf{x}}=\mathbf{T}^{\dagger} \mathbf{y},
$$

where $\mathbf{T}^{\dagger}$ is the generalized inverse of the transform matrix $\mathbf{T}$, named

$$
\mathbf{T}^{+}=\mathbf{P T}^{\mathrm{H}}\left(\mathbf{T P T}^{\mathrm{H}}\right)^{-1} .
$$

$\mathbf{P}$ is the weight coefficient matrix formed by the IDFT of $P_{M V M}$, defining $a_{h}(n)$ to be a Teoplitz matrix as follows

$$
\mathbf{P}=\left[\begin{array}{ccccc}
a_{h}(1) & a_{h}(N-1) & a_{h}(N-2) & \cdots & a_{h}(2) \\
a_{h}(2) & a_{h}(1) & a_{h}(N-1) & \cdots & a_{h}(3) \\
a_{h}(3) & a_{h}(2) & \cdots & \cdots & a_{h}(4) \\
\vdots & \vdots & \cdots & \cdots & \vdots \\
a_{h}(N) & a_{h}(N-1) & \cdots & \cdots & a_{h}(1)
\end{array}\right] .
$$

Considering the singular matrix, regularization can be done to obtain the regularized solution as

$$
\hat{\mathbf{x}}=\mathbf{P T}^{\mathrm{H}}\left(\mathbf{T P T}^{\mathrm{H}}+\rho \mathbf{I}\right)^{-1} \mathbf{y} .
$$

The parameter $\rho$ is the minimum value to make (8) invertible. Let

$$
\mathbf{w}=\left[\begin{array}{llll}
w_{1} & w_{2} & \cdots & w_{L}
\end{array}\right]^{\mathrm{T}}=\left(\mathbf{T P T}^{\mathrm{H}}+\rho \mathbf{I}\right)^{-1} \mathbf{y},
$$

then (8) can be written as $\hat{\mathbf{x}}=\mathbf{P} \mathbf{T}^{\mathrm{H}} \mathbf{w}$, which is a linear combination of the column vector of $\mathbf{P}$. Thus the optimal estimation can be expressed as

$$
\hat{x}(n)=\sum_{k=1}^{L} w_{k} a_{h}\left(n-m_{k}\right) .
$$

By taking DFT, the frequency domain solution can be obtained as

$$
\hat{X}(k)=P_{M V M}(k) \sum_{i=1}^{L} w_{i} e^{-j(2 \pi / N) m_{i} k}=P_{M V M}(k) W(k),
$$

where $W(k)$ is the DFT of a data sequence, which are $w_{k}$ in $\mathrm{n}=m_{k}$ and zeros in other positions.

\section{B. 2-D Case Extension}

SAR imaging is a 2-D data processing. From the model derived in section II, the problem of SAR imaging can be transformed to parameters estimation of 2-D complex sinusoid signal. Thus, the data model of the algorithm in radar imaging processing can be as follows

$$
x(m, n)=\sum_{p=1}^{D} s_{p} e^{j \omega_{p p} m} e^{j \omega_{o p} n}+w(m, n),
$$

where $x(m, n)$ is the data sample after preprocessing in the efficient support domain. The data samples after preprocessing are $m=0, \cdots, L_{1}-1$ and $n=0, \cdots, L_{2}-1$. $\omega_{r p}$ is the range frequency of the scatterer $p . \omega_{a p}$ is the azimuth frequency of the scatterer $p . s_{p}$ is the complex backscatter coefficient of the scatterer $p$. $w(m, n)$ is the additive noise.

According to the 1-D case, the 2-D optimal estimation can be written as follows

$$
\hat{x}(n, m)=\sum_{i=1}^{L_{1}} \sum_{j=1}^{L_{2}} w_{i, j} a_{h}\left(n-m_{i}, m-m_{j}\right),
$$

where $\left\{w_{i, j}\right\}_{i=1 j=1}^{L_{1} L_{2}}$ are the extrapolation coefficients; $a_{h}(n, m)$ is the IDFT of 2-D $P_{M V M}$.

For computing efficiently, the 2-D data matrix should be rearranged to be a vector. Stack the observed data matrix $\mathbf{y}$ and the extrapolated matrix $\mathbf{x}$ in column to be $\operatorname{vec}(\mathbf{y})$ and $\operatorname{vec}(\mathbf{x})$. The same operation can be done to get $\mathbf{P}$ using the IDFT of 2-D $P_{M V M}$. The transform matrix $\mathbf{T}$ can be formed by the position relationship between the observed data and the extrapolated data. Thus the efficient extrapolation solution can be obtained according to the 1-D case.

Though the paper's method and AWNE method both use the minimum weighted norm constraint, AWNE method is an iterative algorithm, and will also iterate the noise and sidelobes, which will lead to miss of targets and artificial targets. The paper's method uses the high resolution spectral estimation without iterative process, which can avoid the data distortion.

\section{RESULTS}

In this section, the performance of the proposed new algorithm is demonstrated via numerical and experimental results.

First, we present a numerical example. The real part of 64 samples observed signal in fig.2 is composed of three complex sinusoids at $0.1,0.19$ and $0.2 \mathrm{~Hz}$ with $10 \mathrm{~dB}$ additive white Gaussian noise, whose amplitudes are 0.5 , 1 and 1, respectively. Fig.2(b) shows the result obtained via FFT over the original 64 point signal padding with zeros to be 512 point, from which it can be noted that the resolution is very poor and sidelobes are very high. Fig.2(c) shows the real part of actual 512 samples signal and (d) is the result of 512 point FFT. After extrapolating (a) using AWNE method with 3 iterations, (e) can be obtained to be 512 point signal and (f) is the result of FFT. The resolution in (f) is improved a lot than that of (b) and sidelobes are also lower. (g) shows the real part of extrapolating signal by the paper's method. The main 
lobe of (h) is much narrower than (f) and sidelobes are even lower, and the main lobe and sidelobes of (h) is close to those of (d), i.e. (h) achieves the performance of actual 512 samples signal.

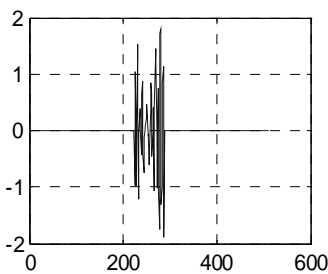

(a)

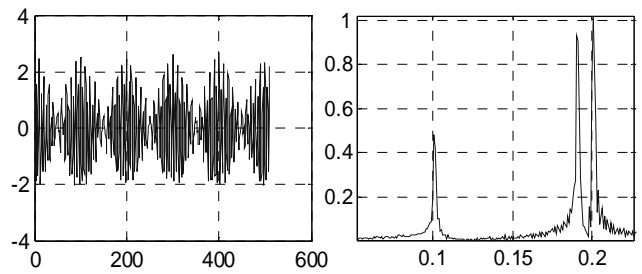

(c)

(d)

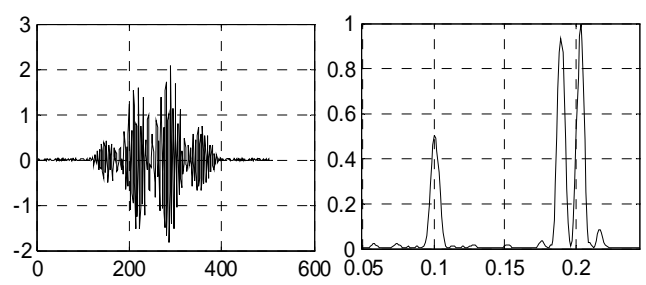

(e)

(f)

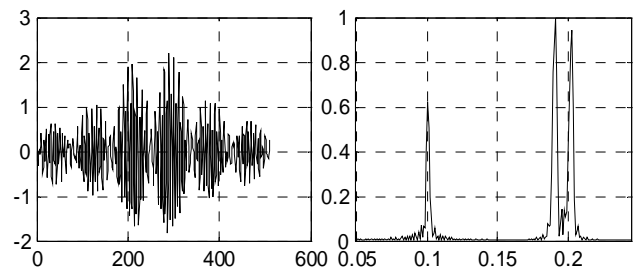

(g) targets unseparated in (b). The more enhancement is shown in (d), which is the imaging result using the paper's method. All of the targets can be separated in (d), which is better than (c).

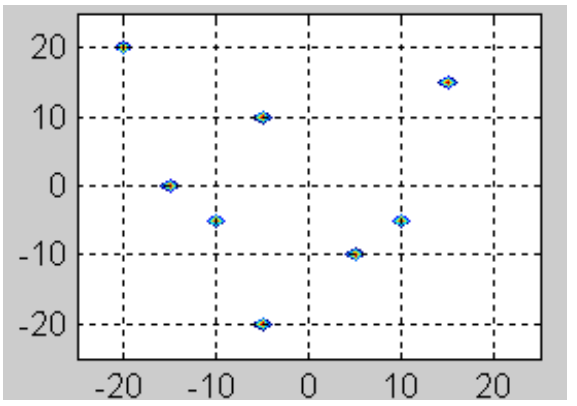

(a)

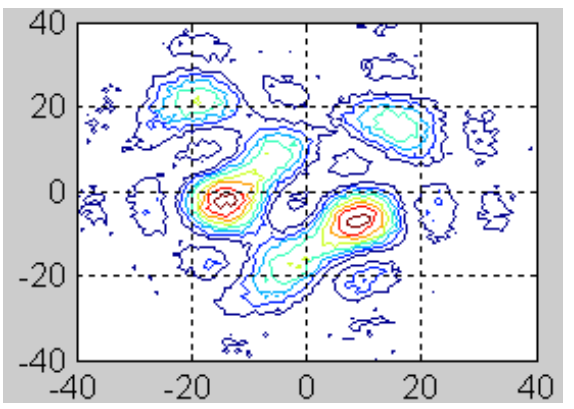

(b)

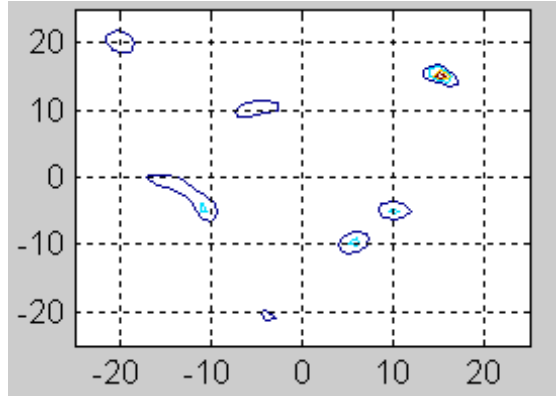

(c)

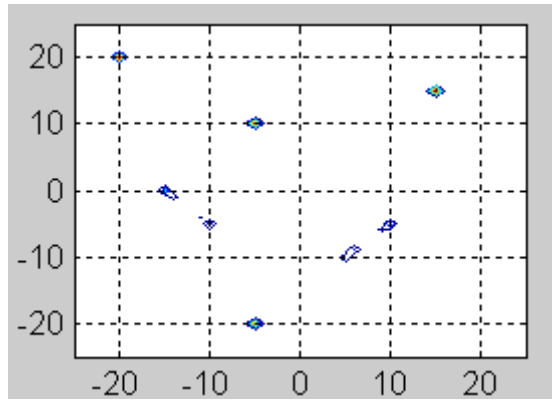

(d)

Fig.3. Simulated point scatters images. (a) The targets' position. (b) Fourier imaging. (c) AWNE. (d) Method in the paper.

The actual calibration SAR data is used to prove the feasibility of the paper's method in actual measured SAR return. From fig. 4 and table I, we can see better result can 
International Archives of the Photogrammetry, Remote Sensing and Spatial Information Sciences, Volume XL-7/W1, 3rd ISPRS IWIDF 2013, 20 - 22 August 2013, Antu, Jilin Province, PR China

be obtained using the paper's method than that of AWNE after 3 iterations, not to mention the RD method.

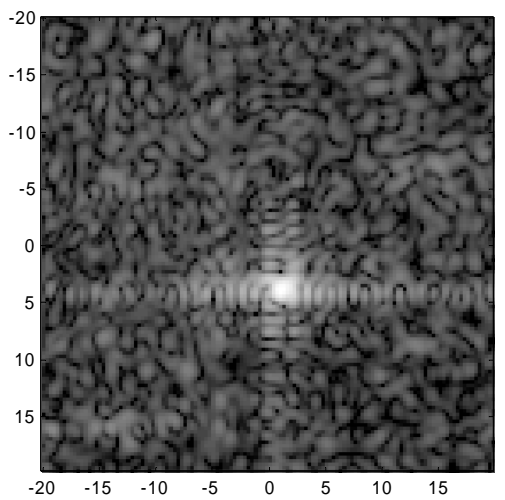

(a)

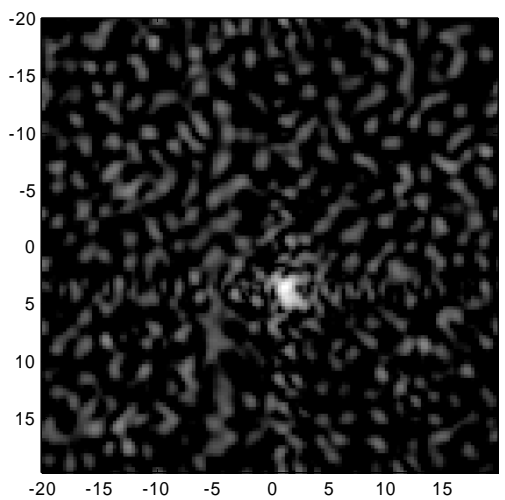

(b)

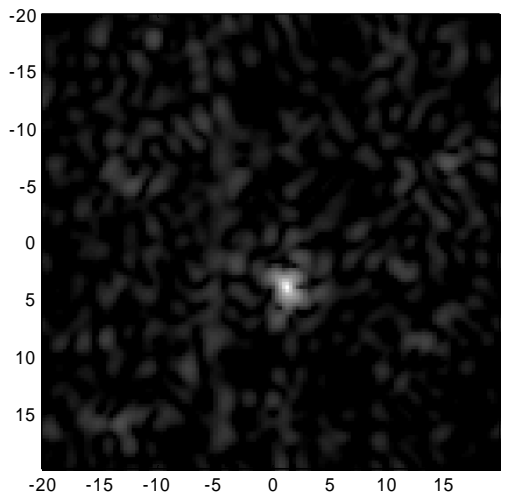

(c)

Fig.4. Actual measured SAR data. (a) RD method. (b) AWNE method. (c) The paper's method.

TABLE I Performance Analysis of Different Methods

\begin{tabular}{|c|c|c|c|}
\hline Performance Index & $\begin{array}{c}\text { RD } \\
\text { Method }\end{array}$ & AWNE & $\begin{array}{c}\text { Paper's } \\
\text { Method }\end{array}$ \\
\hline $\begin{array}{c}\text { Range } \\
\text { resolution(m) }\end{array}$ & 2.5453 & 1.4941 & 0.8385 \\
\hline Range PSLR(dB) & -13.8265 & -25.1351 & -15.1513 \\
\hline Range ISLR(dB) & -15.3648 & -10.9042 & -15.9463 \\
\hline
\end{tabular}

\begin{tabular}{|c|c|c|c|}
\hline $\begin{array}{c}\text { Azimuth } \\
\text { resolution(m) }\end{array}$ & 2.6184 & 1.4029 & 0.7600 \\
\hline Azimuth PSLR(dB) & -12.4227 & -15.1043 & -18.5147 \\
\hline Azimuth ISLR(dB) & -8.8912 & -11.0321 & -15.2571 \\
\hline
\end{tabular}

\section{CONCLUSIONS}

The proposed algorithm combines minimum weighted norm constraint and minimum variance method, which is classified as nonparameter methods and more robust than the parametric approach. The simulated data and actual measured data are both used to show the validation of the algorithm. Compared with AWNE and the Fourier method, the proposed algorithm obtains better results.

\section{ACKNOWLEDGEMENT}

This work is supported by National Natural Science Foundation of China (61001196) and the National High Technology Research and Development Program (Grant Numbers 2011AA120403).

\section{REFERENCES}

Barbarossa, S., Marsili, L., and Mungari, G., 1996, SAR super-resolution imaging by signal subspace projection techniques. in Proceedings of EUSAR'96, Konigswinter, Germany, 267 270.

Brito, A.E., Chan, S.H., and Cabrera, S.D., 1999, SAR Image Formation Using 2D ReWeighted Minimum Norm Extrapolation. in SPIE Conference on Algorithms for Synthetic Aperture Radar Imagery VI, Orlando, Florida, 78-91.

Cabrera, S.D., Flores, B.C., Thomas, G., and Vega-Pineda, J., 1994, Application of One-Dimensional Adaptive Extrapolation to Imrove Resolution in Range-Doppler Imaging. in SPIE's International Symposium on Optical Engineering and Photonics in Aerospace Sensing, International Society for Optics and Photonics, Orlando, FL, 135-145.

Cabrera, S.D., and Parks, T.W., 1991, Extrapolation and spectral estimation with iterative weighted norm modification. IEEE Transactions on Signal Processing. 39(4): 842-851.

Curlander, J.C., and McDonough, R.N., 1991, Synthetic Aperture Radar Systems and Signal Processing. John Wiley \& Sons, Inc.

DeGraaf, S.R., 1998, SAR imaging via modern 2-D spectral estimation methods. IEEE Transactions on 
International Archives of the Photogrammetry, Remote Sensing and Spatial Information Sciences, Volume XL-7/W1, 3rd ISPRS IWIDF 2013, 20 - 22 August 2013, Antu, Jilin Province, PR China

Image Processing. 7(5): 729-761.

Potter, L.C., and Arun, K.S., 1989, Energy concentration in band-limited extrapolation. IEEE Transactions on Acoustics, Speech and Signal Processing. 37(7): 1027-1041. 